\title{
Meta
}

Journal des traducteurs

Translators' Journal

FARINA, A. (2001) : Dictionnaires de langue française du

Canada : Lexicographie et société au Québec, préface de

Claude Poirier, Paris, Honoré Champion, coll. « LEXICA, Mots et

Dictionnaires », $445 \mathrm{p}$.

\section{Éric Poirier}

Volume 48, numéro 3, septembre 2003

Traduction et enseignement

Translation and teaching

URI : https://id.erudit.org/iderudit/007608ar

DOI : https://doi.org/10.7202/007608ar

Aller au sommaire du numéro

Éditeur(s)

Les Presses de l'Université de Montréal

ISSN

0026-0452 (imprimé)

1492-1421 (numérique)

Découvrir la revue

Citer ce compte rendu

Poirier, É. (2003). Compte rendu de [FARINA, A. (2001) : Dictionnaires de langue française du Canada : Lexicographie et société au Québec, préface de Claude

Poirier, Paris, Honoré Champion, coll. « LEXICA, Mots et Dictionnaires », 445 p.] Meta, 48(3), 456-458. https://doi.org/10.7202/007608ar d'utilisation que vous pouvez consulter en ligne. 
FARINA, A. (2001): Dictionnaires de langue française du Canada: Lexicographie et société au Québec, préface de Claude Poirier, Paris, Honoré Champion, coll. «LEXICA, Mots et Dictionnaires», 445 p.

Il est plutôt rare qu'une thèse de doctorat en linguistique soit publiée quasi intégralement, ce qui est pourtant le cas ici et cet exploit mérite d'être signalé. Outre l'originalité de l'étude qui repose sur le portrait global de la lexicographie québécoise et canadienne-française, deux qualités de l'ouvrage expliquent certainement ce choix de la part de la maison d'édition Honoré Champion: le caractère exhaustif de la recension des dictionnaires étudiés ainsi que l'intérêt ethnologique indéniable de l'étude de la société québécoise entreprise sous l'angle de ses productions lexicographiques et des objectifs sous-jacents poursuivis par les lexicographes. Mais avant d'aller plus loin, voici par ordre inverse de présentation, peut-être parce qu'ils retracent ainsi l'ordre chronologique du travail de l'auteure, les principaux éléments de cette riche étude qui nous est proposée.

Signalons tout d'abord les données essentielles de cette étude qui a consisté à dépouiller dans la plupart des dictionnaires publiés au Québec le traitement lexicographique intégral accordé à une quarantaine de mots-clés comme québécisme, bean, bleuet et myrtille, fitter, magasiner, poutine, tsé, week-end et à élaborer pour chacun d'entre eux une fiche historique. Soigneusement choisis pour leur représentativité des différents registres et des caractéristiques lexicales du français d'ici, ces mots-clés et leur traitement lexicographique constituent les assises de l'étude d'Annick Farina. Malheureusement pour le lecteur, on ne trouve en annexe de l'ouvrage que la recension de onze de ces fiches historiques. Il aurait été intéressant (mais peut-être trop lourd les recensions pour la fiche historique du mot-clé char s'étendent sur 17 pages) de publier toutes les fiches historiques qui constituent les éléments de base sur lesquels repose l'analyse de l'auteure. Les fiches font ressortir, ce qui n'est pas rien, toute l'originalité de la contribution de certaines pratiques lexicographiques au fil du temps et des usages de la langue. En plus de permettre de remonter à la source de quelques descriptions curieusement similaires, les fiches illustrent magnifiquement les tribulations du jugement et des partis pris des lexicographes, avec pour toile de fond l'évolution des usages du français québécois depuis 1752 ou quelques décennies plus tard.

En vérifiant le traitement de ces mots-clés dans les dictionnaires de la langue québécoise et en recensant toutes les productions lexicographiques parues au Québec, Annick Farina a entrepris un très imposant chantier bibliographique qui n'est pas sans rappeler celui qu'a réalisé Bernard Quemada sur les dictionnaires français. L'auteure nous propose ainsi une bibliographie chronologique et analytique de tous les dictionnaires québécois, depuis le document manuscrit de 1752 du père Potier jusqu'à la plus récente édition du Dictionnaire des canadianismes de Gaston Dulong, parue en 1999. En outre, comme pour bien souligner le caractère hautement polémique de ces publications et de tout ce qui concerne la description de la langue française au Québec, l'auteure a ajouté une bibliographie portant exclusivement sur les comptes rendus des dictionnaires parus dans la presse québécoise. Un complément d'information utile pour qui s'intéresse, par exemple, à l'accueil réservé aux dictionnaires publiés ici.

Le reste de l'ouvrage se présente sous la forme d'un essai visant à décrire, à partir de l'analyse du traitement des mots-clés choisis, les particularités des dictionnaires 
québécois en regard des courants idéologiques auxquels Annick Farina les associe. Dans sa présentation méthodologique, l'auteure définit quatre intentions dans la production des dictionnaires de langue québécoise: la curiosité, la protection, le combat et la législation qu'elle associe à trois lexicographes types et à trois démarches: le curieux, à la recherche d'un savoir, le puriste, à la recherche d'une pureté, et le lexicographe, à la recherche d'une nationalité. Annick Farina consacre ainsi dans le corps de son ouvrage un chapitre entier de son analyse à chacun de ces types de lexicographes et à leur démarche respective.

Le premier chapitre porte sur les dictionnaires de curieux, qui réunissent aussi bien les ouvrages des glossairistes, ces anciens «chasseurs de perles rares», comme elle les décrit, que les travaux des linguistes et universitaires modernes qui collectionnent les «mots en marge». Dans le travail de ces curieux, anciens et modernes, comme c'est le cas dans certains traitements du mot tuque, Annick Farina dégage des problèmes méthodologiques inhérents aux premiers pas de la lexicographie d'ici, mais aussi, ce qui est plus pesant, de la difficile tâche des lexicographes québécois d'aujourd'hui, entre les glossairistes de la première heure, qui versent parfois dans la mythification de la langue qu'ils souhaitent décrire, et les puristes, qui cherchent à «purifier» la langue d'ici, au risque d'y trouver parfois aussi un «entre-langue» qui, bien entendu, n'existe pas, ni en Amérique, ni ailleurs.

Le deuxième chapitre porte justement sur les publications de ces puristes, que l'auteure appelle les éducateurs, et leurs dictionnaires de combat. Elle note les différentes formes de publications qu'ils privilégient: des recueils prescriptifs du type «Dites ceci, mais ne dites pas cela», jusqu'à la très volumineuse collection des Cahiers de l'Office de la langue française à laquelle appartient, par exemple, la fameuse série des fascicules sur le vocabulaire de l'automobile. L'auteure passe en revue les conditions d'attribution et de traitement lexicographique des anglicismes, des impropriétés et des canadianismes. Par exemple, elle observe avec perspicacité le statut singulier d'un verbe comme fitter qui est présenté au mieux comme un intrus manifeste auquel on n’accorde généralement aucune marque ni aucune description précise.

Dans le troisième et dernier chapitre de son ouvrage, qui porte sur la période qui s'est écoulée depuis la Révolution tranquille, Annick Farina analyse l'évolution des mentalités linguistiques québécoises dans la reconstruction d'une langue et d'une culture, avec notamment ses hésitations (Louis-Alexande Belisle), ses excès (Léandre Bergeron), ses innovations (le Dictionnaire du français Plus) et son ethnocentrisme (l'adaptation du Robert avec le Dictionnaire québécois d'aujourd'hui). Comme l'explique l'auteure, ces mouvements témoignent du malaise bien réel des lexicographes québécois qui ne demandent qu'à «redevenir de plein droit francophone, en restant québécois "à part entière" et non "de part en part" ".

En conclusion, Annick Farina préconise une description lexicographique qui s'appuie sur les réussites et les échecs du passé, qui s'adresse ainsi à un lecteur ayant dépassé le stade de l'« adolescence linguistique », à la fois réaliste et pragmatique, sans penchant exagéré pour l'écrit ou pour l'oral, pour la science ou pour le populisme, pour les différences et pour les points communs avec le français en France et avec les autres variétés de français. Au fond, l'auteure nous invite à réfléchir sur le travail fondamental des lexicographes d'ici et d'ailleurs et sur l'équilibre fragile entre l'œuvre, les désirs de l'auteur et les moyens dont il dispose, et aussi entre l'œuvre et 
les besoins et exigences des utilisateurs. En la suivant dans cette réflexion sur la méthode lexicographique, on ne peut s'empêcher de conclure qu'elle gagne en lexicographie un très beau pari de la linguistique, auquel rêvent d'ailleurs toujours les linguistes générativistes et cognitivistes, celui d'accéder à l'universel par le truchement indispensable du particulier.

Éric Poirier

Université de Montréal, Montréal, Canada

Balliv, C. (2002): Les traducteurs transparents. La traduction en France à l'époque classique, Bruxelles, Éditions du Hazard, collection Traductologie, 240 pages.

À l'instar du $\mathrm{xv}^{\mathrm{e}}$ siècle italien (Quattrocento) et du $\mathrm{xvI}^{\mathrm{e}}$ espagnol (Siglo de Oro), le $\mathrm{XVII}^{\mathrm{e}}$ siècle français constitue sans conteste, tout en étant bien français, un des points forts absolus dans l'Histoire de la Culture Occidentale et de la Culture Universelle. Par sa vigueur extraordinaire (on ne l'appelle pas sans raison le Siècle des Querelles), par la diversité de ses excellences et surtout par la qualité exceptionnelle de ses représentants, le siècle de Louis XIV s'impose naturellement à tout qui se montre intéressé par l'étude diachronique d'un domaine particulier du savoir et du savoir-faire humain. Ce fut le temps où « un duc de la Rochefoucauld, l'auteur des Maximes, au sortir de la conversation d'un Pascal et d'un Arnauld, allait au Théâtre de Corneille» (Voltaire).

Dans son ouvrage sur la traduction en France à l'époque classique, le professeur C. Balliu poursuit un triple but: étudier la traduction du règne de Louis XIV dans un cadre de globalité politique, économique, religieuse, civile, juridique et artistique; réfléchir sur la traductologie en tant que dimension et développement linguistiques et littéraires (la traduction comme activité linguistique et littéraire) et, surtout, analyser la spécificité intellectuelle de la traduction (traductologie). En effet, on a pratiquement tout dit sur le $\mathrm{XVII}^{\mathrm{e}}$ siècle, sauf peut-être sur la traduction. Or, dans la réalité, tous les aspects de la traduction y ont été mis à l'honneur, la pratique d'abord, mais aussi l'enseignement, la réflexion et le débat.

Sur le plan théorique, le professeur Balliu, partisan convaincu de Georges Mounin (il lui emprunte notamment l'image de la transparence), considère que le débat littéralité vs fidélité est essentiellement et avant tout un faux débat. Il n'y a pas d'incompatibilité (antinomie) (p. 23) entre beauté et fidélité. Au Xvir siècle, écrivains et traducteurs se rencontrent et parlent le même langage. Que se soit à l'Académie ou dans les salons. Même l'opposition entre Académie et Jansénisme n'est qu'un leurre (p. 23-24).

L'approche du professeur Balliu est en même temps chronologique et thématique. En partant de Claude de Seyssel, le grand traducteur de Louis XII, d'Etienne Dolet, le «premier» traductologue français, de l'opposition Marot-du Bellay, du traducteur Vaugelas et de Jacques Amyot (le legs du passé) il débouche finalement sur Antoine Galland, le traducteur des Mille et une Nuits (1704) qui clôture le Grand Siècle. À l'intérieur de celui-ci l'auteur analyse attentivement l'état de la traduction par rapport à la qualité diachronique de la langue française, la préciosité, la réflexion traductologique, la querelle des Anciens et des Modernes, Port-Royal et l'enseignement ('’́cole des enfants de langues). 